\title{
The Design and Implementation of Traffic Accident Identification System Based on Video
}

\author{
Chenwei Xiang ${ }^{1}$, Tuo Wang ${ }^{2}$
}

\begin{abstract}
With the rapid growth of the count of vehicles, congestion and traffic accidents frequently occur. The situation triggers a series of social problems. The application research on computer vision and digital image processing system is presented in this paper provides a real-time method which identify traffic accident and manage the traffic accident more convenient, accurate and efficient .The system also offer the technologies to analysis the traffic accident management information, traffic accident rescue and responsibility determination.
\end{abstract}

Keywords: computer vision, intelligent transportation system, traffic accident identification, video, traffic accident management

\section{Introduction}

In recent years, with the rapid development of economic and social progress, the road has been accessible everywhere, the number of cars is increasing, and then traffic problems are increasing, traffic congestion and traffic accidents occur frequently. According to statistical data, the number of national road traffic accidents involving casualties is 210812 in 2011 one year. It's a current problem to be solved focused by our country and oversea that is how to establish an effective intelligent transportation system (intelligent transportation system, ITS) which can achieve real-time traffic scene monitoring, scheduling and control. In

\footnotetext{
${ }^{1}$ C. Xiang $(\square)$

The School of Electronic and Information Engineering, Xi'an Jiaotong University,710049,Xi’an, Shaanxi, P.R.China

e-mail:weierxiaoxie@126.com

${ }^{2}$ T.Wang $(\bowtie)$

The School of Electronic and Information Engineering, Xi'an Jiaotong

University,710049,Xi'an, Shaanxi, P.R.China

e-mail: twang@sei.xjtu.edu.cn
} 
the system, we should identify the traffic accident at first and deal with information about the traffic accident. This paper designs and implements the traffic accident identification system which is based on video.

\section{Design of The Traffic Accident Identification and Management System}

The system is divided into two management levels, municipal and district levels. In accordance with the way that different districts deal with the accidents occur in own district. The system will automatically identify the traffic accident and sent back the video of the accident to the image library of district command monitoring center the accident road belongs. The system will also classify the alarm by the severity of the traffic accidents, and display the rank on monitoring terminal. The staff in district command monitoring center check and manage the alarms quickly according to the severity of traffic accidents. When traffic accident exceeds the limit level, the system will report to the municipal command monitoring center, and municipal command monitoring center checks the accident and guide treatment for traffic accident remedial work. Municipal command monitoring center only transfer the information and related videos from district command monitoring center.

\subsection{Architecture Design of the Traffic Accident Identification and Management System}

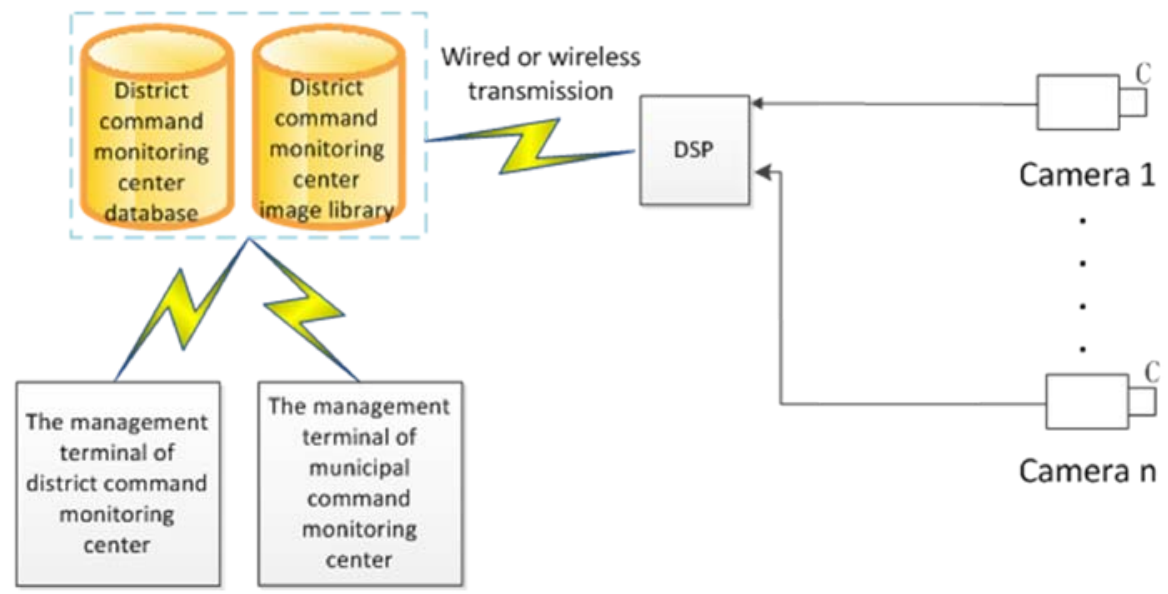

Fig .2.1.system components figure 
The hardware of the system is shown in figure.2. 1. The cameras capture the videos, related DSP analysis and identify traffic accident, when the traffic accident is identified, the video of it will be sent to the district command monitoring center the road belongs by wireless or wired devices. Municipal command monitoring center transfer the desired videos from district command monitoring center's image library.

In the hardware of the system, the great computing power of DSP can identify the traffic accident in this road quickly and accurately. Therefore, several cameras may be divided into a set corresponds to a workstation to complete the automatic traffic accident identification. The accident-prone road can use DSP program.

\subsection{Function Design of Traffic Accident Identification and Management System}

The system consists of communication, information storage, traffic accident identification and traffic accident management four subsystems, each subsystem's main functions are as follows:

Communication subsystem: Subsystem transfers the video captured by cameras to the DSP or workstation, then DSP or workstation compute the video and sent the analysis result to district command monitoring center. And the system also completes the communication between municipal and district command monitoring center.

Information storage subsystem: subsystem storages the original video of traffic accident, the level, the place, specification information dealing with the aftermath of the traffic accident, the rescue hospital and so on.

Traffic accident identification subsystem: Subsystem identifies the traffic accident, intercepts the video information including the accident process and identifies the level.

Traffic accident management subsystem: Subsystem alarms on the screen of the district command monitoring center after identifying traffic accidents, and then displays the alarms' profiles by the level. The staff clicks the profiles to display the related traffic accidents' videos and determines responsibilities. It also can quarry the history video of the traffic accidents or statistic the traffic accidents data by road, time and level. In order to ensure data completeness, subsystem backups the videos, or takes the videos in secondary storage (hard drive) into the system.

System software features are shown in Fig .2.2. 


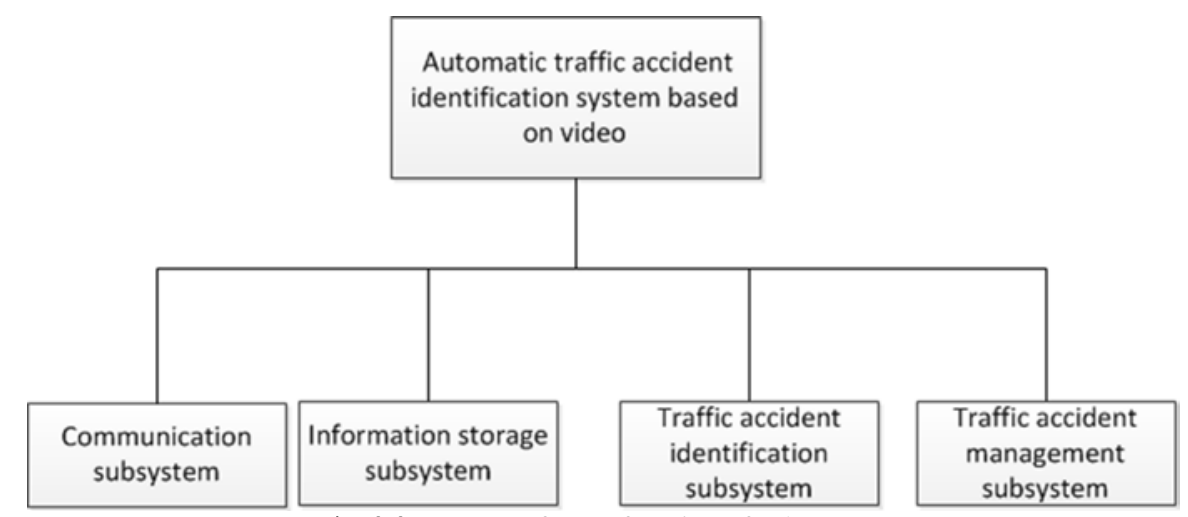

Fig .2.2 System software function of subsystems

\section{Algorithm and Key Technologies of the Traffic Accident Identification}

\subsection{Architecture Design of the Traffic Accident Identification and Management System}

The environment of highway is different from urban road. This paper is based on the highway traffic accident. The environment of highway is easier, when vehicle is running, less interference on the road. The vehicle generally damage badly after the traffic accident.

System processes the input video frame by frame, build the background model and segment the vehicles from it. Then system detects the vehicle stopping and detects the vehicle's outer contour curve, the outer contour curve compares with the contour curve in database. If the result proves that outer contour curve changes a lot, it shows a collision of the vehicle. If the outer contour curve has no change, it may be the part of the vehicle has been damaged. The system should detect the part feature, resulting in the traffic accident level and other information.

\subsection{Key Technologies}

The key technologies in the system are the background modeling based on color space, the vehicle's feature extraction and description based on the outer contour and the part of the vehicle's feature extraction and description based on improved SIFT. 


\subsubsection{Background Modeling Based on Color Space}

We propose an approach to building the road real-time model based on spatial clustering (SC). In this approach, we abstract the background modeling as the process of spatial clustering in time axis.

From statistical point of view, in a location of an image the background pixels' color value is fluctuating in a range. Therefore, we do clustering analysis for these pixels to form a number of data clusters. Objects in one cluster are similar with each other, while objects between clusters are different. Every cluster represents one possibility of the background model. In a color image, each pixel can be regarded as a point in $3 \mathrm{~d}$ space, whose position is together determined by the color components that constitute this point. The choice of the rules according to which these points are clustered is the key that the background model obtained is accurate or not. In this article, we use this color distortion rule and brightness distortion rule as the clustering criterion of road background modeling[1].

On this basis, we will design the algorithm as follows:

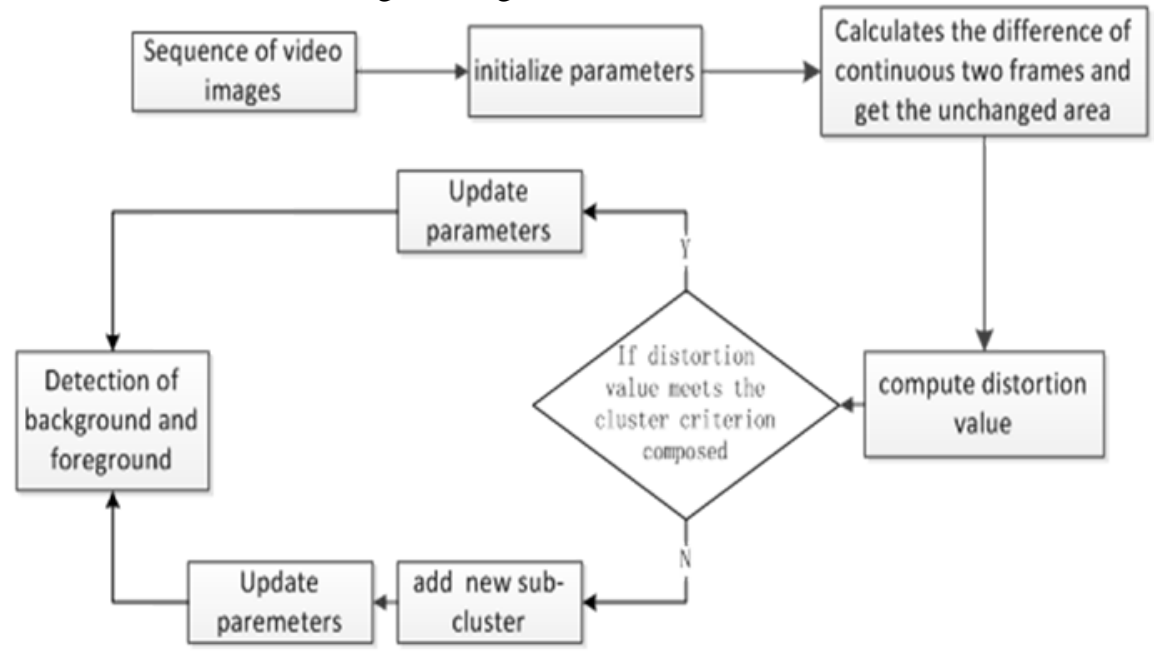

Fig .3.1 the algorithm flowchart

The detailed algorithm is according to the literature [2]. 

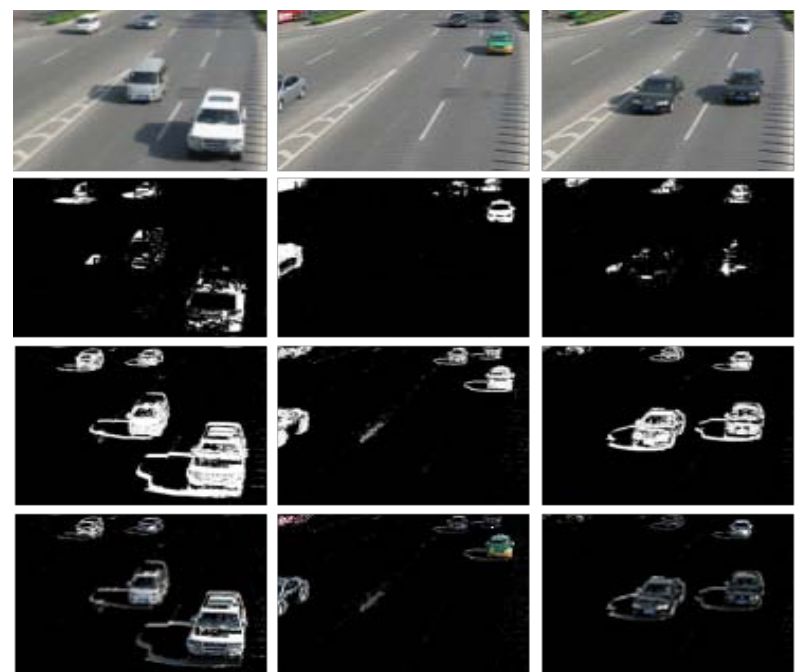

Fig.3.2. Parts of results. The top row shows the original images in traffic scenes, the second row are results obtained by using non-parametric Bayesian modeling, the third row are results obtained by using proposed approach, the fourth row masked original images obtained by using proposed approach. Morphological method is not used in the experiments.

\subsubsection{The Vehicle' s Feature Extraction and Description Based on the Outer Contour}

The extracted foreground image is converted to binary image, filtering and extracting the edge, then using Douglas Peucker algorithm(DP algorithm)[3]to achieve the edge feature extraction of vehicle. Specific steps are as follows:

(1) Between the endpoints of the curve A and B to make a straight line segment $\mathrm{AB}$, the line segment is the string of the original curve;

(2) Get the maximum distance from the original curve point $\mathrm{H}$ to the $\mathrm{AB}$ and calculate its distance called h;

(3) Compare the present threshold value $\mathrm{X}$ with $\mathrm{h}$, if $\mathrm{h}<\mathrm{X}$, use $\mathrm{AB}$ to approximate the original curve, end the approximation work;

(4) If $\mathrm{h}>=\mathrm{X}$, the original curve divide into two parts by $\mathrm{H}, \mathrm{AH}$ and $\mathrm{BH}$ and then carry out the step (1) (2) (3) for the two curves;

(5) When all curves are dealt with, connect all the points in the approximation to get a line chart, end the approximation work. The points are the feature points of vehicle.

Since the scale of the transformation influences seriously, the, this paper hope to the extracted corner points is not affected by the image scale. To address this situation, we can replace the original coordinate feature information for the vector feature information. The corner feature of the vehicle expressed in vector form, the centroid of the target is used as the original point. In this way, we can effectively overcome the defect caused by the scale transformation when we 
detect corner points. In order to unify features, the vectors are normalized so that the value of the elements in the interval $[0,1]$.

\subsubsection{The Part of the Vehicle' s Feature Extraction and Description Based on Improved SIFT}

In the vehicle recognition progress, we need to search and match the extracted feature points in database. As the SIFT feature vector is 128-dimensinal, its high dimensional lead to the search efficiency is extremely low. In order to ensure the rotation invariance of the descriptor in SIFT algorithm, we assign a main direction for the feature point and rotate axes according to the main direction before generating descriptors. If the descriptors itself has a good rotational invariance, this step can be omitted. As round has good rotational invariance, this paper draws on ideological division round, using a circular neighborhood instead of a matrix neighborhood to generate the 64-dimensinal feature descriptor [4-5]. It's shown as Fig.3.3.

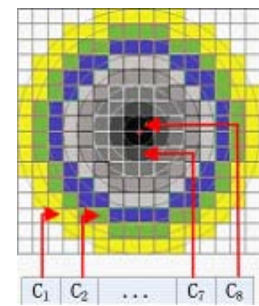

Fig.3 .3 Round Neighborhood

The improved algorithm has two advantages:(1)Compared with the division based on matrix neighborhood, the division based on round neighborhood can assure rotational invariance, eliminates the step calculating the main direction and rotating the axes.(2)Different points in different areas has its own value, the weight of the points is higher if it's close to the feature point ,the Gaussian weighting before the gradient magnitude value calculating, because the Gaussian contour lines is the concentric center which center is feature point, the step Gaussian-weighted can combine with calculating feature vector in improved algorithm.

Collecting the two dimensional images of the vehicle from different directions, extract features from the images to make combinations of feature descried, final generate 3D feature database of the vehicle, we can identify the kind of vehicle via searching the matching the feature database. In order to facilitate research, this paper's work applies in horizontal plane. The longitude angle has no change, rotate the camera in latitude plane capturing images.

It is a key point to build multi-angle three-dimensional database that how to choice the change angle, it should ensure the feature will has less missing and no more redundancy. If the angle changing value $\beta$ is too large, too much feature lost. If $\beta$ is too small, there are a lot of redundant points have high similarity. Through 
the statistical analysis from the result of experiments, $20^{\circ}$ is the best change angle. We search and match the features between two adjacent angles, the repeated features retain only once in database, in order to reduce data redundancy and retain the feature information more completely. In the vehicle identification, system need to compare vehicle images from videos with the vehicle features in database, the accuracy of feature points directly affects the vehicle identification is correct or not.

\section{Conclusion}

This paper presents a traffic accident identification system based on video, designs the architecture of system and studies the related algorithms. The experiments show that the algorithms are feasible, but the algorithms are not realtime, it needs to research and improve the algorithms further in future.

\section{Acknowledgement}

This work is supported by the National Natural Science Foundation of China (No. 61071217.).

\section{References}

1. Kim K, Chalidabhongse T H, Harwood D, Davis L S(2004) Background modeling and subtraction by codebook construction.In: Proceedings of International Conference on Image Processing. Singapore, Singapore: IEEE, 3061-3064.

2. Chenwei X,Tuo W et al(2013) Roads modeling using the method of color spatial clustering.In: Journal of Image and Graphics,8(18):985-990.

3. Douglas D H, Peucker T K(1973) Algorithms for the reduction of the number of points required to represent a line or its caricature. In: The Canadian Cartographer, 10(2) : 112-122.

4. Mikolajczyk K, Schmid C(2005) A performance evaluation of local descriptors[J]. In:IEEE Transactions on Pattern Analysis and Machine Intelligence, 27 (10): 1615-1630.

5. Hua LQ,Xu W et al(2013)Vehicle recognition using improved SIFT and multi-view model.In: Journal of $\mathrm{Xi}^{\prime}$ an Jiaotong University,4(47):92-99. 